\title{
Asymptotic Giant Branch Stars in the Nearby Dwarf Galaxy Leo P*
}

 \\ A. A. Zijlstra ${ }^{5}$, A. S. Hirschauer ${ }^{1}$ (D), E. D. Skillman ${ }^{7}$ (D), and S. Srinivasan ${ }^{8}$ \\ ${ }^{1}$ Space Telescope Science Institute, 3700 San Martin Drive, Baltimore, MD 21218, USA; sgoldman@ @stsci.edu \\ ${ }^{2}$ University of Texas at Austin, McDonald Observatory, 2515 Speedway, Stop C1402, Austin, TX 78712, USA \\ ${ }^{3}$ Rutgers University, Department of Physics and Astronomy, 136 Frelinghuysen Road, Piscataway, NJ 08854, USA \\ ${ }^{4}$ Department of Physics and Astronomy, University of North Carolina Chapel Hill, Chapel Hill, NC 27599-3255, USA \\ 5 Jodrell Bank Centre for Astrophysics, Alan Turing Building, University of Manchester M13 9PL, UK \\ ${ }^{6}$ Lennard-Jones Laboratories, Keele University, ST5 5BG, UK \\ ${ }^{7}$ Minnesota Institute for Astrophysics, School of Physics and Astronomy, 116 Church Street S.E., University of Minnesota, Minneapolis, MN 55455, USA \\ ${ }^{8}$ Instituto de Radioastronomía y Astrofísica, Universidad Nacional Autónoma de México Antigua Carretera a Pátzcuaro \#8701 Ex-Hda. San José de la Huerta \\ Morelia, Michoacán. C.P.58089, México \\ Received 2019 August 7; revised 2019 August 31; accepted 2019 September 3; published 2019 October 21
}

\begin{abstract}
We have conducted a highly sensitive census of the evolved-star population in the metal-poor dwarf galaxy Leo P and detected four asymptotic giant branch (AGB) star candidates. Leo $\mathrm{P}$ is one of the best examples of a nearby analog of high-redshift galaxies because of its primitive metal content ( $2 \%$ of the solar value), proximity, and isolated nature, ensuring a less complicated history. Using medium-band optical photometry from the Hubble Space Telescope (HST), we have classified the AGB candidates by their chemical type. We have identified one oxygen-rich source which appears to be dusty in both the HST and Spitzer observations. Its brightness, however, suggests it may be a planetary nebula or post-AGB object. We have also identified three carbon-rich candidates, one of which may be dusty. Follow-up observations are needed to confirm the nature of these sources and to study the composition of any dust that they produce. If dust is confirmed, these stars would likely be among the most metal-poor examples of dust-producing stars known and will provide valuable insight into our understanding of dust formation at high redshift.
\end{abstract}

Unified Astronomy Thesaurus concepts: Asymptotic giant branch stars (2100); Dwarf galaxies (416); Circumstellar dust (236); Metallicity (1031); Stellar mass loss (1613); Late stellar evolution (911); Mira variable stars (1066); Extreme carbon stars (512); Carbon stars (199)

\section{Introduction}

In the advanced stages of their evolution, low- to intermediatemass stars $\left(0.8 \lesssim M \lesssim 8 M_{\odot}\right)$ become important producers of dust. Once in the thermally pulsing phase of the asymptotic giant branch (TP-AGB), these stars can vary dramatically in brightness on timescales between 60 and 2000 days as a result of pulsations, which are a critical component of how they produce dust. Their variable nature allows circumstellar gas to be levitated to large radii near the gravitational barrier, where it cools and condenses into dust and is then driven away by radiation pressure (e.g., Höfner \& Olofsson 2018 and references therein). As they produce dust, TP-AGB stars become obscured in the optical and bright in the infrared (IR), as stellar light is scattered and absorbed by the dust and re-radiated in the IR.

AGB stars can have surface chemistries rich in either oxygen (M-type) or carbon (C-type), which toward their later stages of evolution result in the production of either oxygen-rich or carbonrich dust grains. AGB stars that are lower mass or are early in their evolution are oxygen-rich stars. As they evolve, their surface chemistry is dictated by the initial mass and metallicity, which affect the efficiency and timescales of internal processes. Third dredge-up events cause AGB stars above $\sim 1.3-2 M_{\odot}$ to become carbon stars, while even more massive AGB stars $\left(\sim 3-5 M_{\odot}\right)$ will become carbon stars and then transition back to oxygen-rich stars

\footnotetext{
* Based on observations made with the NASA/ESA Hubble Space Telescope, obtained at the Space Telescope Science Institute, which is operated by the Association of Universities for Re- search in Astronomy, Inc., under NASA contract NAS 5-26555. These observations are associated with program HSTGO-14845.
}

as a result of hot-bottom burning (Boothroyd et al. 1993). The mass limit ranges are a result of these internal processes. The upper and lower mass limits of carbon stars decrease in more metal-poor samples (Marigo et al. 2017).

From the ground, we can observe molecules like $\mathrm{TiO}$ and $\mathrm{VO}$ on the surfaces of these stars. From space, however, we can detect additional molecules in the IR with much more sensitivity, like water in oxygen-rich AGB stars and $\mathrm{C}_{2}+\mathrm{H}_{2}$ and $\mathrm{HCN}$ in carbonrich AGB stars (Aoki et al. 1999; Cernicharo et al. 1999; Volk et al. 2000; Kraemer et al. 2002), which allow for categorization of chemical type. We can use them to gain insight into the efficiency of the dust production rate of TP-AGB stars, their dust properties, composition, and stellar evolution, and to reveal distinct chemical populations.

\subsection{Dust Production and Metallicity}

Dust reservoirs have been observed in galaxies and quasars out to redshifts $z \sim 6$ (Bertoldi et al. 2003; Robson et al. 2004; Beelen et al. 2006). The origin of this dust, however, remains unclear. Massive oxygen-rich TP-AGB stars are expected to be able to produce dust $30 \mathrm{Myr}$ after forming (for a $10 M_{\odot}$ star), compared to at least 280-380 Myr for carbon stars (Volk et al. 2000; Ventura et al. 2002; Herwig 2004). The dust production of massive oxygen-rich TP-AGB stars should be affected by metallicity as these stars should not be able to create the raw materials needed to seed the nucleation of dust grains, as opposed to carbon stars (Lagadec \& Zijlstra 2008; Nanni et al. 2018). As a result, the earliest and highest-redshift dust producers (the massive 
oxygen-rich TP-AGB stars) are expected to be limited by their initial metallicity.

Observations of nearby carbon-rich TP-AGB samples conflict on the degree that metallicity affects dust production (van Loon 2006; van Loon et al. 2008; McDonald et al. 2011b; Sloan et al. 2012, 2016). For oxygen-rich AGB stars, studies have shown that dust production (Sloan et al. 2008, 2010) and expansion velocity (Marshall et al. 2004; Goldman et al. 2017) are limited in metal-poor AGB stars in nearby galaxies and globular clusters. This, however, is only the case for AGB stars. Supernovae ( $\mathrm{SNe}$ ) may dominate dust production at high redshift and in metal-poor galaxies, yet the level of dust that they destroy (both newly formed and pre-existing) remains unclear (Lakićević et al. 2015; Temim et al. 2015).

\subsection{Metal-poor AGB Samples}

The largest available samples of evolved stars more metalpoor than the Milky Way lie in the Large and Small Magellanic Clouds (LMC, SMC), at around one-half and one-fifth solar metallicity, respectively. Smaller samples have been detected that span a range of metallicities in nearby dwarf galaxies and globular clusters. We require larger samples and in even more metal-poor environments to study the effect of metallicity on the evolution of AGB stars and their contribution of dust to the interstellar medium (ISM).

Dwarf galaxies. Metal-poor AGB populations have been detected in nearby galaxies spanning 2 dex in metallicity (Battinelli et al. 2007; Battinelli \& Demers 2008; Menzies et al. 2008, 2010, 2011, 2015; Whitelock et al. 2009; Lorenz et al. 2011; McDonald et al. 2012; Sloan et al. 2012; Whitelock 2012; Bellazzini et al. 2014; Boyer et al. 2015b, 2017; Jones et al. 2018). One of these sources is in the Sagittarius Dwarf Irregular Galaxy (Sag DIG), with a gas-phase metallicity of $12+\log (\mathrm{O} / \mathrm{H})=7.26-7.50$ (Saviane et al. 2002), and was found to have a pulsation period of 950 days (Whitelock et al. 2018), indicative of a very late stage of evolution and a high progenitor mass, and was additionally found to be oxygen-rich and dusty (Boyer et al. 2017). The DUST in Nearby Galaxies with Spitzer (DUSTiNGS; Boyer et al. 2015a) and Spitzer InfraRed Intensive Transients Survey (Kasliwal et al. 2017) were designed to look for dusty metal-poor, long-period variables in the IR and have successfully uncovered populations in nearby galaxies (Boyer et al. 2015a, 2015b; Goldman et al. 2019; Karambelkar et al. 2019). Additional observations with the Hubble Space Telescope (HST) have chemically classified a significant fraction of these (Boyer et al. 2017). These observations have revealed candidate AGB stars in seven galaxies reaching down to metallicities of $[\mathrm{Fe} / \mathrm{H}]=-1.85$.

Globular clusters. Variables have also been detected in globular clusters within the Galaxy (Whitelock 1986; Clement et al. 2001; Feast et al. 2002; Origlia et al. 2002; Lebzelter \& Wood 2005; Boyer et al. 2006; Matsunaga \& IRSF/SIRIUS Team 2007; McDonald et al. 2009, 2011a, 2011b) and SMC (Feast et al. 2002). Metal-poor, dust-producing AGB stars have also been found in $\omega$ Cen (McDonald et al. 2011c), a cluster with an average metallicity of $[\mathrm{Fe} / \mathrm{H}] \sim-1.6$ dex (Smith et al. 2000). These are low-mass $\left(<1 M_{\odot}\right)$ AGB stars, however, and not representative of the high-mass TP-AGB stars that would contribute dust in high-redshift galaxies.

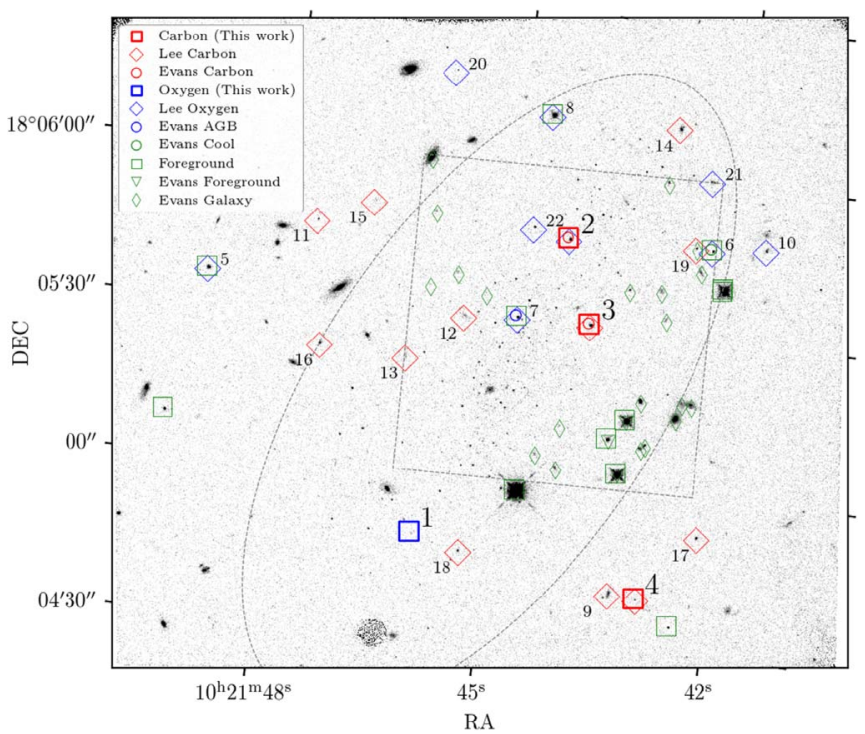

Figure 1. Hubble Space Telescope (HST) F127M medium-band drizzled mosaic of Leo $\mathrm{P}$ (half-light radius shown with the gray dashed oval) with sources categorized as oxygen-rich (blue) and carbon-rich (red). Our AGB classifications as well as those made by Lee (2016) and Evans et al. (2019) are shown with different shapes, where the label "Evans AGB" corresponds to the source with a confirmed membership to Leo P using Ca II absorption (Source 22), and "Evans Cool" (Source 7) corresponds to the source that was only confirmed as a cool star from Ca II absorption. Green symbols show sources that have been categorized as foreground stars or background galaxies in this work and Evans et al. (2019). The footprint of the previous Very Large Telescope Multi Unit Spectroscopic Explorer observation by Evans et al. (2019) is shown as the gray dashed square and the Gemini North Near InfraRed Imager observations cover the whole field of view as well as the Spitzer and HST observations. The oval artifact at the bottom of the image is a known HST artifact.

\subsection{Leo $P$}

To search for high-redshift AGB analogs we have observed the nearby dwarf galaxy Leo $\mathrm{P}$ (shown in Figure 1), which is the most metal-poor, gas-rich galaxy resolvable with current instruments. Leo $\mathrm{P}$ is a dwarf galaxy with a stellar mass of $5.7 \times 10^{5} M_{\odot}$ and distance of $1.62 \pm 0.15 \mathrm{Mpc}$ (McQuinn et al. 2013, 2015a). Observations have shown that the ratio of gas to stars is $2: 1$ and the ratio of total mass to baryonic mass is 15:1 (Bernstein-Cooper et al. 2014). Originally discovered by the Arecibo Legacy Fast ALFA survey (Giovanelli et al. 2005), observations have revealed the galaxy's stellar populations (McQuinn et al. 2015a) as well as a luminous star-forming H II region (Rhode et al. 2013). One of the most remarkable aspects of Leo P, however, is its ISM metallicity $(12+\log (\mathrm{O} / \mathrm{H})=$ $7.17 \pm 0.04$; Skillman et al. 2013), which is essentially identical to that of the well known low-metallicity galaxy I Zw 18 (e.g., Skillman \& Kennicutt 1993), but is 10 times closer. The low metallicity is also evident in the stellar population at $1.8 \%$ solar $([\mathrm{Fe} / \mathrm{H}]=-1.8 \pm 0.1 \mathrm{dex}$; McQuinn et al. 2015a).

Leo $\mathrm{P}$ has shown a nearly constant star formation rate (SFR) over the last 4 Gyr. Over the last 200 Myr the SFR of Leo P has been $\sim 6.5 \times 10^{5} M_{\odot} \mathrm{yr}^{-1}$ (McQuinn et al. 2015a), making it the most metal-poor star-forming galaxy in the Local Volume. Searching for AGB stars in star-forming galaxies is critical, as the short evolutionary timescales of the massive dust-producing AGB stars are limited to galaxies with recent star formation. Using the color-magnitude diagram (CMD)-fitting tool $\mathrm{MATCH}$ (Dolphin 2002), we have fit a synthetic CMD of Leo $P$ 
Table 1

Leo P Observations



Note. The field of view (FOV) of the IRAC detector has two chips separated by 1.52 .

and calculated the expected number of massive AGB stars $\left(M>5 M_{\odot}\right)$ to be around three. This code has the added benefit of randomly sampling the initial mass function, which is important for galaxies with low SFRs like Leo P.

Leo $\mathrm{P}$ has been shown to lie near to the luminositymetallicity and stellar mass-metallicity relationships for dwarf galaxies. Unlike all of the other extremely metal-poor (XMP) galaxies like I Zw 18 (Berg et al. 2012), however, Leo P is not offset from the luminosity-metallicity relation (Skillman et al. 2013). This suggests that Leo P's star formation history (SFH) is more similar to a high-redshift galaxy than any other stellar sample resolvable with current instruments. Leo $\mathrm{P}$ is also the only known XMP dwarf galaxy that lacks a complicated history, which allows us to better constrain the different processes that can affect the abundances of metals in dwarf galaxies. Based on the age-metallicity relationship of Rafelski et al. (2012), Leo $P$ has abundances representative of a galaxy with a redshift of $z \gtrsim 3.2$, or a galaxy in an epoch $11.7 \mathrm{Gyr}$ ago. While Leo $\mathrm{P}$ differs from high-redshift galaxies in its lowermass evolved star population and enrichment history, it is an excellent environment in which to study the effects of metallicity on stellar evolution and dust production.

In this paper we present the results from a highly sensitive census of the evolved star population in the metal-poor dwarf galaxy Leo P. We have detected four AGB candidates, two of which show evidence of dust. One of these dusty sources may be a planetary nebula (PN) or post-AGB star that has been producing oxygen-rich dust. The other dusty source is likely a dusty carbon star. Section 2 describes the observations and methods used to identify the AGB candidates. Section 3 presents the AGB candidates, compares them with previous observations, and discusses how Leo $\mathrm{P}$ compares to a highredshift galaxy.

\section{Observations and Methods}

Observations included imaging using both Spitzer and the HST to isolate AGB stars by their chemical type in the near-IR, and determine whether they are producing significant dust in the IR. Table 1 provides details of the observations. The HST observations occurred just 10 days after the Spitzer observations, minimizing issues with stellar variability, since TP-AGB stars can vary by $>1$ mag at near-IR wavelengths over $\sim 300-1000$ days. Table 2 shows the photometry from our observations as well as archival observations. The HST observations were sensitive enough to detect stars 3 mag below the tip of the red giant branch (TRGB), and the sensitivity of our Spitzer observations begins to fall off after [4.5] $18 \mathrm{mag}$ (Figure 2).

\subsection{Spitzer Imaging}

Leo P was observed using Spitzer in the IRAC bands at 3.6 and $4.5 \mu \mathrm{m}$. We have performed point-spread function (PSF) photometry on the dithered exposures using the DUSTiNGS pipeline (Boyer et al. 2015a), which uses DAOphot II and ALLSTAR (Stetson 1987). The observations consisted of four exposures using the 16-point spiral medium dither pattern. For one source (Source 1; described further in Section 4), the $3.6 \mu \mathrm{m}$ counterpart was not originally detected by the DUSTiNGS photometry pipeline. To overcome this, we performed aperture photometry with a $2^{\prime \prime}$ aperture on both the 3.6 and $4.5 \mu \mathrm{m}$ images to determine the [3.6]-[4.5] color $(\approx 0.5 \mathrm{mag})$. We then applied this color to the PSF-derived $4.5 \mu \mathrm{m}$ magnitude to obtain a $3.6 \mu \mathrm{m}$ magnitude.

\subsection{HST Imaging}

Leo $\mathrm{P}$ was imaged in the near-IR with HST's Wide Field Camera 3 IR channel (WFC3/IR), using the F127M, F139M, and F153M filters with pivot wavelengths of $1.27,1.38$, and $1.53 \mu \mathrm{m}$, respectively ${ }^{9}$ (program GO-14845). These filters allow for the disentanglement of the carbon- and oxygen-rich AGB stars (e.g., Boyer et al. 2017). A four-position box dither pattern was employed to mitigate imaging artifacts and increase the spatial resolution, with a total exposure time of $\sim 800 \mathrm{~s}$ for F127M and F153M and $\sim 850 \mathrm{~s}$ for F139M, which has a slightly lower throughput. The detector was read out using the WFC3/IR STEP sequence, which samples the detector with the shortest possible sequence for the first few reads, then samples logarithmically thereafter to provide a high dynamic range for fields containing both bright and faint sources. Figure 1 shows a mosaic image using the F127M filter data.

We performed PSF photometry using DOLPHOT's WFC3specific module (Dolphin 2000). A drizzled mosaic of the F127M images was used as the reference image (created using HST Drizzlepac v2.0) to allow forced photometry of faint objects in the individual images. To limit contamination from background galaxies and foreground sources, we implemented cuts for sharpness and crowding in all three bands: $\left(\Sigma \text { Sharp }_{\lambda}\right)^{2}<0.5$ and $\left(\Sigma \mathrm{Crowd}_{\lambda}\right)^{2}<5$ plus an additional constraint in the $\mathrm{F} 127 \mathrm{M}$ band: Sharp $\mathrm{F} 127 \mathrm{M}>-0.025$ and Crowd $_{\mathrm{F} 127 \mathrm{M}}<1.5$. By restricting the sharpness parameter, we minimize contamination from extended objects and cosmic rays. The crowding restriction eliminates objects that are significantly affected by nearby objects. We visually inspected all objects of interest to confirm that they are truly unblended point sources.

\footnotetext{
9 The DOI for this data set as well as the wide-band ACS:HST optical data from McQuinn et al. (2015a) is 10.17909/t9-67a4-t896.
} 
Table 2

TP-AGB Candidates Identified Here and in the Literature

\begin{tabular}{|c|c|c|c|c|c|c|c|c|c|c|c|c|c|c|}
\hline ID & $\begin{array}{l}\text { R.A. } \\
\text { (J2000) }\end{array}$ & $\begin{array}{l}\text { Decl. } \\
\text { (J2000) }\end{array}$ & $\begin{array}{c}\mathrm{F} 475 \mathrm{~W} \\
(0.48 \mu \mathrm{m}\end{array}$ & $\begin{array}{c}\text { F814W } \\
(0.81)\end{array}$ & $\begin{array}{c}J \\
(1.25)\end{array}$ & $\begin{array}{c}K \\
(2.2)\end{array}$ & $\begin{array}{c}\text { F127M } \\
(1.27)\end{array}$ & $\begin{array}{c}\text { F139M } \\
(1.39)\end{array}$ & $\begin{array}{c}\text { F153M } \\
(1.53)\end{array}$ & [3.6] & [4.5] & $\begin{array}{l}\text { Type } \\
\text { Lee }\end{array}$ & $\begin{array}{l}\text { Type } \\
\text { Evans }\end{array}$ & Type \\
\hline 1 & 155.441598 & 18.080108 & 24.73 & 24.09 & $\ldots$ & $\ldots$ & 24.05 & 23.43 & 22.54 & 20.79 & 20.27 & $\ldots$ & $\ldots$ & $\mathrm{M}$ \\
\hline 2 & 155.434403 & 18.096345 & 24.10 & 20.88 & 19.46 & 18.45 & 19.47 & 19.12 & 19.14 & 18.40 & 18.07 & M & $\mathrm{C}$ & $\mathrm{C}$ \\
\hline 4 & 155.428871 & 18.077750 & $\ldots$ & $\ldots$ & 22.62 & 19.69 & 23.00 & 21.87 & 21.43 & 18.15 & 18.57 & $\mathrm{C}$ & $\ldots$ & $\mathrm{C}$ \\
\hline 5 & 155.454186 & 18.093011 & $\cdots$ & $\cdots$ & 18.95 & 18.21 & 19.05 & 18.99 & 18.83 & 17.96 & 17.96 & M & $\cdots$ & $\mathrm{K} / \mathrm{FG}$ \\
\hline 6 & 155.426428 & 18.096447 & 24.13 & 21.82 & 20.97 & 20.25 & 20.83 & 20.63 & 20.38 & 19.94 & 19.63 & M & $\mathrm{AGB} / \mathrm{K}$ & $\mathrm{K} / \mathrm{FG}$ \\
\hline 9 & 155.430410 & 18.077854 & $\ldots$ & $\ldots$ & 20.41 & 18.94 & $\ldots$ & $\ldots$ & $\ldots$ & $\ldots$ & $\ldots$ & $\mathrm{C}$ & $\ldots$ & Ext \\
\hline 10 & 155.423518 & 18.096696 & 23.27 & 21.83 & 21.02 & 19.86 & $\ldots$ & $\ldots$ & $\ldots$ & $\cdots$ & $\cdots$ & M & $\cdots$ & Ext \\
\hline 11 & 155.448452 & 18.096050 & $\cdots$ & $\ldots$ & 21.42 & 19.69 & $\cdots$ & $\cdots$ & $\ldots$ & 18.35 & 17.92 & $\mathrm{C}$ & $\cdots$ & Ext \\
\hline 12 & 155.439832 & 18.091725 & 26.39 & 24.98 & 21.64 & 18.81 & $\ldots$ & $\cdots$ & $\ldots$ & $\ldots$ & $\ldots$ & $\mathrm{C}$ & $\ldots$ & $?$ \\
\hline 13 & 155.442865 & 18.089298 & 24.67 & 23.93 & 21.59 & 20.10 & $\ldots$ & $\ldots$ & $\ldots$ & $\ldots$ & $\ldots$ & $\mathrm{C}$ & $\ldots$ & Ext \\
\hline 14 & 155.428950 & 18.102686 & 24.43 & 22.18 & 20.31 & 18.69 & $\ldots$ & $\ldots$ & $\ldots$ & 17.29 & 17.13 & $\mathrm{C}$ & $\ldots$ & Ext \\
\hline 19 & 155.427389 & 18.096439 & 24.62 & 22.53 & 21.48 & 20.03 & $\ldots$ & $\ldots$ & $\ldots$ & 19.63 & 19.41 & $\mathrm{C}$ & Gal & $?$ \\
\hline 20 & 155.441592 & 18.104558 & $\ldots$ & $\ldots$ & 20.52 & 19.48 & $\ldots$ & $\ldots$ & $\ldots$ & $\ldots$ & $\ldots$ & M & $\ldots$ & $?$ \\
\hline 21 & 155.426829 & 18.100057 & 24.14 & 22.68 & 19.98 & 19.04 & $\ldots$ & $\ldots$ & $\ldots$ & $\cdots$ & $\cdots$ & M & $\cdots$ & Ext \\
\hline 22 & 155.436432 & 18.096797 & 23.96 & 22.04 & 21.20 & 20.31 & 21.24 & 21.02 & 20.85 & $\ldots$ & $\cdots$ & M & $?$ & Ext \\
\hline
\end{tabular}

Note. AGB candidates discovered in this work, Evans et al. (2019), and Lee (2016). For sources with HST data, coordinates have been corrected using Gaia astrometry $(\Delta \alpha=-0$." 41, $\Delta \delta=-0$ ". 53). For sources without $H S T$ data, coordinates are from Gemini (Lee et al. 2006). Photometric data are shown in Vega magnitudes; F475W and F814W are from McQuinn et al. (2015a); $J$ and $K$ are from Lee (2016). IDs are the same as source numbers used within the text. Our chemical classifications as well as those determined by Lee (2016) and Evans et al. (2019) are shown in the last three columns. Sources with classifications but no photometry were cut in either the crowding, sharpness, or TRGB cuts. Sources labeled as K/FG were classified as likely K-type giants or foreground stars; sources labeled Ext are classified as extended sources and likely background galaxies.

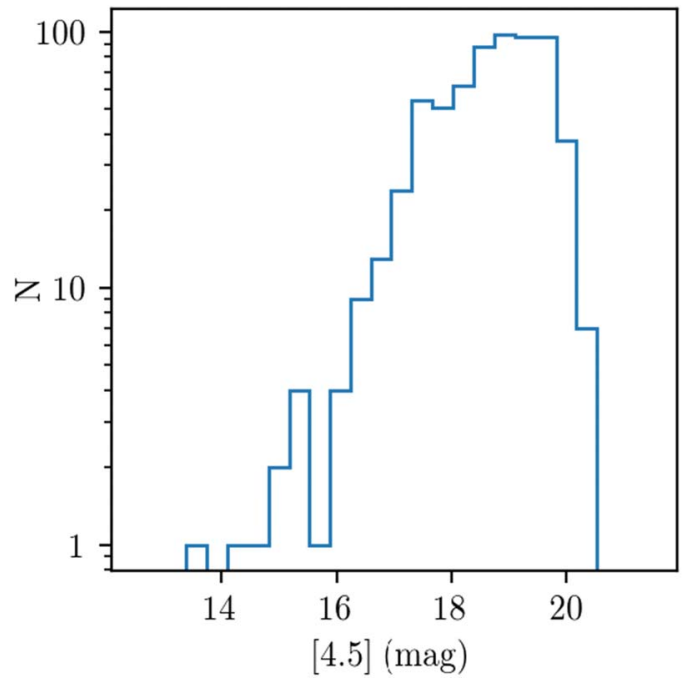

Figure 2. Luminosity function of the Spitzer [4.5] photometry. The histogram shows a drop in sensitivity below $\sim 18$ mag.

The measured reddening toward Leo $\mathrm{P}$ is $E(B-V)=0.0227$ mag (Schlafly \& Finkbeiner 2011) and, assuming $R_{\mathrm{V}}=3.1$, this corresponds to an extinction $A_{\mathrm{V}}=0.0693 \mathrm{mag}$. The photometry in each of the $H S T$ filters was corrected for this interstellar extinction using the corrections from Cardelli et al. (1989), an approximate shift of -0.016 mag. The Spitzer data were uncorrected because the amount of extinction was smaller than the photometric uncertainties in the IRAC filters.

\subsection{Previous Observations}

Previous studies have used the Gemini North Telescope and the European Southern Observatory's Very Large Telescope (VLT) to search for evolved stars in Leo P. These programs have detected 11 oxygen-rich AGB candidates and 15 carbonrich AGB candidates (Lee 2016; Evans et al. 2019).

Gemini North-NIRI. Lee (2016) used the Near InfraRed Imager and Spectrometer (NIRI; Hodapp et al. 2003) on the Gemini North Telescope to target AGB stars using the $J$ and $K$ bands and determine their chemical type. The $J-K$ color probes circumstellar extinction. These bands are not, however, as effective for determining the chemistry of more evolved dusty TP-AGB stars, as molecular features within the $J$ waveband make AGB stars redder in their $J-K$ color. Lee (2016) identified nine oxygen-rich and 13 carbon-rich AGB candidates.

VLT-MUSE. Evans et al. (2019) obtained optical spectra with the VLT's Multi Unit Spectroscopic Explorer (MUSE; Bacon et al. 2010). These observations cover the $483-930 \mathrm{~nm}$ wavelength range, which includes $\mathrm{TiO}$ and $\mathrm{C}_{2}$ molecular bands and the Ca II triplet. $\mathrm{C}_{2}$ Swan bandheads at 5165 and $5636 \AA$ were detected in two AGB candidates, confirming their carbon-rich chemical type. Two additional sources show the Ca II triplet in absorption, where it was used to measure a recessional heliocentric velocity in one of these sources (Source 7; $262 \pm 8 \mathrm{~km} \mathrm{~s}^{-1}$ ) that was consistent with membership of Leo P. The second source (Source 22) was only detected in one Ca II band, and may be either a foreground star, K-type giant, or an AGB star in Leo P. 


\subsection{Determining AGB Chemistry and Isolating Dusty AGB Candidates}

In order to isolate the evolved AGB population of Leo P, we limited our sample using cuts in magnitude and F127M - F153M color. The dust surrounding TP-AGB stars obscures them in optical bands and increases their F127M-F153M color. We thus only considered sources with F127M-F153M color typically redder than the RGB following the reddening vector, or sources above the TRGB $\left(M_{\mathrm{F} 127 \mathrm{M}}<21 \mathrm{mag}\right.$; Boyer et al. 2017) as possible AGB candidates. We also considered one source to the left of the RGB where oxygen-rich stars have been shown to show deep water absorption (Figure 5 in Boyer et al. 2019).

Boyer et al. $(2013,2017)$ showed that the HST WFC3/IR F127M, F139M, and F153M filters separate AGB stars by their chemical type (Figure 1 from Boyer et al. 2017). These filters probe wavebands that are affected by different molecular features: $\mathrm{H}_{2} \mathrm{O}$ for oxygen-rich $\mathrm{AGB}$ stars and $\mathrm{CN}+\mathrm{C}_{2}$ for carbon stars. Alongside the stellar evolutionary models, in the F127M-F139M and F139M-F153M color-color space (Figure 3), our sources clearly separate into two populations: oxygen- and carbon-rich.

\section{Results and Discussion}

We have successfully identified four AGB candidates and classified them by chemical type. We have also shown that the method of AGB classification of carbon stars using HST medium-band filters is robust even at extremely low metallicities; this remains to be confirmed in our dusty AGB candidates. Table 2 shows photometry from HST, Spitzer, and NIRI, as well as our classification and the classifications by Lee (2016) and Evans et al. (2019). Sources with classifications but no HST photometry were cut in the crowding, sharpness, or TRGB cuts, and are not likely to be AGB stars (see Section 2). Figure 4 shows HST/ACS F814W mosaic snapshots of our AGB candidates. Our sample of AGB candidates is similar in number to those found in other galaxies of similar size to Leo $\mathrm{P}$ $\left(M_{V}=-9.3 \mathrm{mag}\right)$, for example Phoenix $\left(M_{V}=-9.9 \mathrm{mag}\right)$ or Sag DIG $\left(M_{V}=-11.5 \mathrm{mag}\right)$ with one and three sources, respectively (Menzies et al. 2008; McConnachie 2012; Whitelock et al. 2018). Of the four sources detected, two appear dusty. This is consistent with the estimated number of AGB stars predicted using MATCH $\left(N_{\mathrm{AGB}} \sim 3\right)$.

\subsection{Contaminant Sources}

Searches for evolved stars in the near-IR and optical are commonly affected by contaminating sources, typically foreground stars or K-type giants. With the angular resolution of the $H S T$ (and using sharpness cutoffs) we are able to identify and remove any extended sources such as background galaxies visually. For the point-like sources, we can identify stars that may be either foreground stars or warmer giants and supergiants by their position in color-color space (shown in Table 3 and Figures 3 and 8 (below)); results from the Trilegal simulations have confirmed foreground stars with these near-IR colors (Boyer et al. 2017). Point sources may also be young stellar objects (YSOs), PNe, or post-AGB stars. These phases are typically much shorter than the AGB, and thus we expect contamination from these sources to be rare. Of the 25 sources that passed the photometry and TRGB cutoffs, we have classified four as AGB candidates, nine as extended sources, and 12 as either foreground
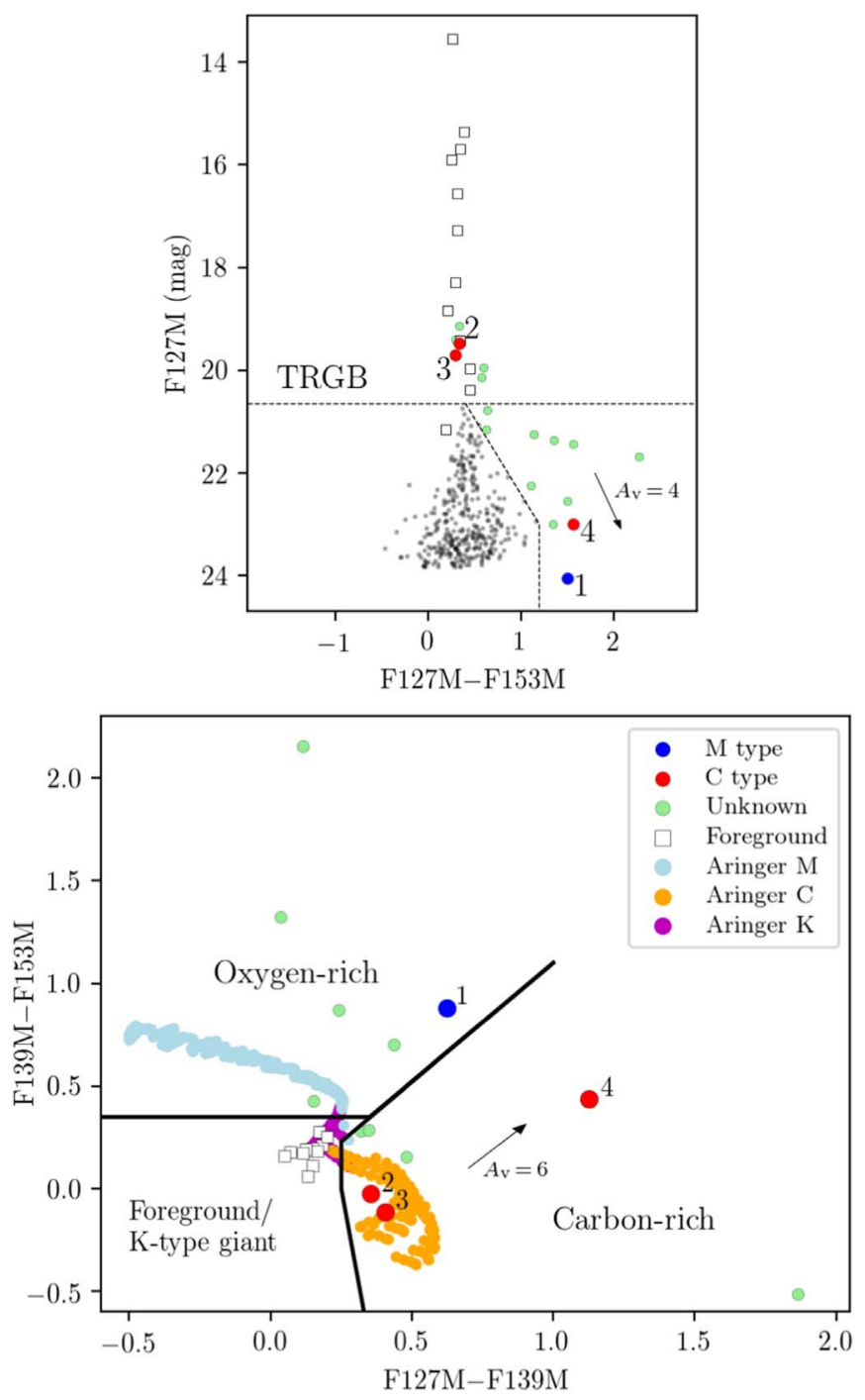

Figure 3. HST color-magnitude and color-color diagrams showing (left) sources above the tip of the red giant branch or dusty enough to be considered AGB candidates and (right) a utilization of the method successfully used by Boyer et al. (2013, 2017, 2019) to disentangle carbon-rich (C-type; red) and oxygen-rich (M-type; blue) evolved stars, and sources classified as either foreground stars or K-type giants (white squares); colors apply to both diagrams. Also shown are stellar models from Aringer et al. $(2009,2016)$ as well as extinction vectors showing reasonable values for $A_{\mathrm{V}}$, which assume an $R_{\mathrm{V}}=3.1$ and the extinction law from Cardelli et al. (1989). While the extinction law applies to interstellar dust, this level of obscuration will have a similar effect on AGB dust. Sources that meet our TP-AGB selection criteria, but are visibly extended or not point-source-like in the HST images (shown in the Appendix), are labeled as "Unknown" (green).

stars or K-giants. This was done in part by inspecting the shape of the source within the image. Without high-resolution imaging, many of the extended background galaxies would have been classified as evolved stars. Confirmation of these classifications as well as our AGB candidates will require spectroscopic follow-up.

Red supergiants (RSGs) could also possibly contaminate our sample. These sources, while rarer than AGB stars, may be mistakenly classified as luminous oxygen-rich AGB candidates. Within the SMC at one-fifth solar metallicity, RSGs have been shown to have warmer median temperatures (Levesque et al. 2006; Massey et al. 2007). It is also expected that a decrease in metal content will result in a decrease in opacity and thus an increase in surface brightness. Given these assumptions, any 




Source 4 (right) are expected to be dusty.

RSGs should lie closer to the foreground sources in our colorcolor diagram (Figure 3). Given the low brightness of our oxygen-rich candidate, we do not expect it to be an RSG.

\subsection{Oxygen-rich AGB Candidate}

We have detected one oxygen-rich AGB candidate in Leo $P$ (Source 1). Figures 3 and 5 show the source's position in near-IR CMDs. While we expect that the star is obscured and fainter in the HST wavebands as a result of dust, the Spitzer photometry $([4.5]=20.27 \mathrm{mag})$ is also fainter than what is expected for a TP-AGB star. The source lies around two magnitudes below the oxygen-rich AGB stars found in the SAGE-LMC sample at $4.5 \mu \mathrm{m}$. We used the Dusty Evolved Star $\mathrm{Kit}^{10}$ (DESK), a PYTHON package for fitting the spectral energy distributions (SEDs) of AGB stars with a range of model grids using a least-squares fit. The source was fitted with a grid of DUSTY models that use grains from Ossenkopf et al. (1992). These models, however, do not fit all of the HST filters well (Figure 6). The source is unlikely to be a YSO as it is far from any nearby star-forming regions. This source may not be a massive O-rich TP-AGB star, but rather a post-AGB star or PN. If this is the case, the source may still be showing that silicate dust can form at these metallicities. Another possibility is that the source is a background galaxy; however, the full width at half maximum of the source is within the range of other confirmed nearby stars. Spectroscopic follow-up is needed to confirm the nature of this source.

\subsection{Carbon-rich AGB Candidates}

We have detected three carbon-rich AGB candidates in Leo $\mathrm{P}$, including one that may be dusty. The two sources that appear dustless based on their Spitzer and HST colors (Sources 2 and 3) lie above the TRGB and were also previously categorized as carbon-rich AGB candidates by their strong $\mathrm{C}_{2}$ Swan bandheads at 5165 and $5636 \AA$ (Evans et al. 2019). Figure 7 shows the SEDs of Sources 2, 3, and 4, along with radiative transfer models from the dust-growth code of Nanni et al. (2018), fit with the DESK. Shown is a best-fitting model of Source 3. Dustier models fit poorly, because the dust shifts too much emission into the IR. The photospheric CO absorption and poor fit with dusty models suggest that Sources 2 and 3 are carbon-rich AGB stars with little to no dust.

The dusty candidate (Source 4) has a uniquely shaped SED that begins to fall in brightness longwards of $4 \mu \mathrm{m}$ (Figure 7). Carbon stars tend to have bluer [3.6]-[4.5] colors as a result

\footnotetext{
${ }^{10}$ https://github.com/s-goldman/Dusty-Evolved-Star-Kit
}

of $\mathrm{CO}$ absorption at 4-6 $\mu \mathrm{m}$. This feature gets veiled with increasing dust (Frogel \& Hyland 1972; Boyer et al. 2011; Blum et al. 2014). Source 4 has a [3.6]-[4.5] color of $-0.42 \mathrm{mag}$, indicative of $\mathrm{CO}$ absorption. The medium-band $H S T$ colors also suggest a source that is heavily obscured by dust (F127M-F139M = $1.12 \mathrm{mag})$. It may be the case that the source is not dusty enough to be veiled by the continuum dust emission, yet still shows signs of heavy dust obscuration in the optical. Another alternative is that this source may have a unique geometry that reveals both the dust emission and photosphere of the source.

It is also possible that Source 4 is not an AGB star but in an entirely different phase of evolution. It is located outside of the half-light radius of the galaxy (Figure 1). While AGB stars have previously been discovered in the outskirts of dwarf galaxies (McQuinn et al. 2017), fewer of them are expected at these distances. AGB stars at these distances may possibly indicate a "bursty" SFH that causes star formation to occur more in the outskirts of the galaxy at later times. This would then create younger populations to mix with older populations (inside-out galaxy formation; El-Badry et al. 2016).

\subsection{Comparison with Previous Detections}

We have independently recovered the two carbon-rich AGB candidates previously identified spectroscopically (Sources 2 and 3). Our two new dusty AGB candidates (Sources 1 and 4) were found outside of the footprint of the previous MUSE observations and may have been too faint to be detected in the broadband near-IR imaging with NIRI.

We have also detected the two oxygen-rich AGB candidates found by Evans et al. (2019, Sources 7 and 22), which fall in the "foreground/K-type giant" area of our color-color diagram (Figure 3). While only one of these sources has a confirmed membership to Leo P (Source 22), neither source was found to have significant $\mathrm{TiO}$ bands in their optical spectra, and we do not see evidence of $\mathrm{H}_{2} \mathrm{O}$ in our more recent observations. In metal-poor environments it is expected that the lower natal abundance of oxygen results in warmer atmospheres and a higher fraction of K-type giants that do not produce dust.

The additional candidates only found by Lee (2016) may be highlighting the limitations of using broadband and lowresolution optical photometry to identify and classify AGB stars. These bands are affected by molecular absorption as well as both carbon- and oxygen-rich dust, making these bands less reliable for categorizing and classifying dusty AGB stars. We have determined that 10 of their 21 candidates are extended, and four are either foreground stars or K-type giants. 




Figure 5. IRAC CMD showing our oxygen (blue circle) and carbon (red circles) AGB candidates in Leo $\mathrm{P}$, along with the locations of other spectroscopically confirmed classes of LMC stars from SAGE-SPEC (Jones et al. 2017). Shown are AGB stars (solid lines), PNe (dashed lines), post-AGB stars (dotted-dashed lines), background galaxies (gray circles), and remaining SAGE-SPEC sources (background points). A COLIBRI isochrone using the Nanni et al. (2013, 2014, 2016) dust growth models with a log(age in years $)=8.8$, LMC metallicity, and a normalized number of seed particles $\log \epsilon_{\mathrm{S}}=-13$, is also shown in cyan.

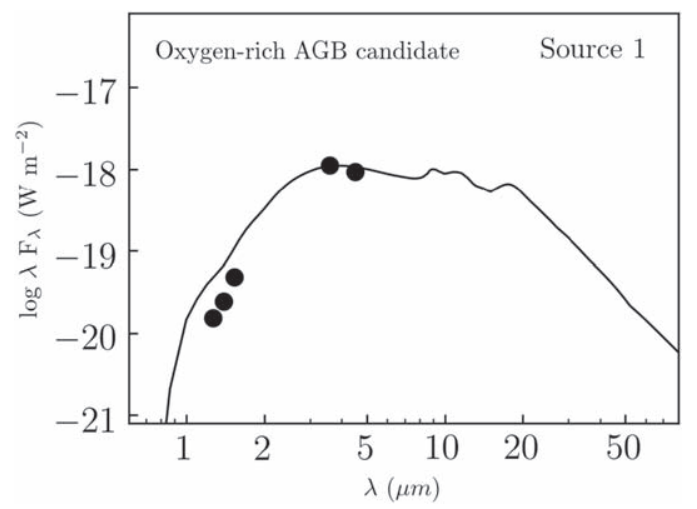

Figure 6. Spectral energy distribution of the oxygen-rich AGB candidate, shown with Spitzer and HST photometry, as well as a DUSTY radiative transfer model (Elitzur \& Ivezić, 2001) that uses a blackbody and grains from Ossenkopf et al. (1992), fitted using the Dusty Evolved Star Kit.

\subsection{Leo $P$ as an Analog of a High-redshift Galaxy}

Leo P's isolation makes it a useful analog for high-redshift galaxies. Being unaffected by galaxy interactions or stripping events (ram pressure or tidal) ensures that we have a better understanding of its history. Due to their longer evolutionary timescales, low-mass AGB stars may be limited in their number in high-redshift galaxies, as opposed to Leo P. Galaxies out to a redshift of $\sim 8$, however, will have had enough time for both oxygen- and carbon-rich AGB stars to form and produce dust (Sloan et al. 2009 and references therein). As Leo P is less massive, it is also expected to have a smaller stellar population, making it more difficult to catch shorter phases of evolution like the AGB.

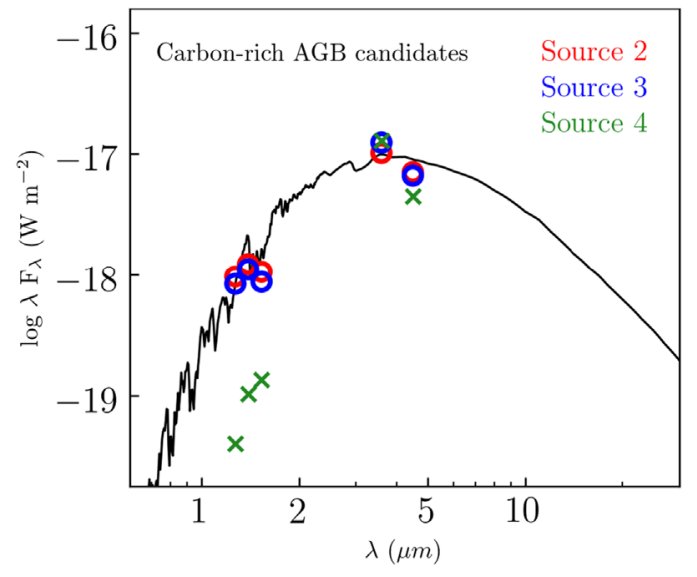

Figure 7. Spectral energy distributions of the carbon-rich AGB candidates, shown with Spitzer and HST photometry, as well as models from the dust growth code from Nanni et al. (2018). Shown is the best-fitting model of Source 3 using an LMC carbon model (solid black) which uses dust grains from Hanner (1988).

In terms of their chemical abundances, Leo $\mathrm{P}$ has only retained 5\% of its SNe ejecta (McQuinn et al. 2015b), limiting the abundance of r-process elements, which can be important sites of dust nucleation. While carbon stars are expected in both environments, the difference in the amount of carbon present may differ. Additional carbon in either environment may lock up more oxygen in $\mathrm{CO}$, limiting the ability of the oxygen-rich AGB stars to produce dust. The strength of this effect at these metallicities, however, is not well known. Understanding the impact of metallicity on dust production on the AGB will require observing additional metal-poor samples in galaxies like I Zw 18 or Leoncino (AGC 198691; Hirschauer et al. 2016).

\section{Conclusion}

We have conducted a census of the population of evolved stars in the metal-poor dwarf galaxy Leo P. This galaxy is the most metal-poor, gas-rich galaxy that we can resolve with current instruments and is an excellent environment for studying stellar populations similar to those in high-redshift galaxies. Our census has yielded four AGB candidates. They were selected on the basis of their near-IR medium-band photometry. We have found most of the AGB candidates previously detected by low-resolution imaging to be extended objects and likely background galaxies.

The one oxygen-rich candidate and one of the carbon-rich candidates appear to be dusty in both the Spitzer and HST data, while the other two carbon-rich candidates appear dust-free. Our oxygen-rich AGB candidate shows high levels of obscuration in the HST bands, and a reddened color in the Spitzer bands, indicative of dust. The low luminosity in these bands may be the result of metallicity effects or changes in dust properties, but is more likely to suggest that the source is in another phase of evolution (e.g., post-AGB, PN). Given the small mass of Leo $\mathrm{P}$ and the short length of these phases, this source is exceptionally rare. If the source is a dust-producing AGB star, however, it would likely dominate the dust production within Leo $\mathrm{P}$. The dusty carbon-rich candidate appears to be highly obscured in the HST data ([1.27] $-[1.53]=1.55 \mathrm{mag})$, but does not show reddened Spitzer colors. The source has a blue [3.6]-[4.5] color of $-0.42 \mathrm{mag}$, 
likely from strong $\mathrm{CO}$ absorption at $4.5 \mu \mathrm{m}$, which is commonly found in carbon stars. The heavy extinction in the HST bands may also arise from a unique geometry.

These candidates may allow us to better understand dust production in metal-poor environments and high-redshift galaxies, but this requires follow-up in the IR. Spectroscopic follow-up with the James Webb Space Telescope will allow us to both confirm the nature of these sources and study how dust forms at these metallicities $\left(Z \sim 2 \% \mathrm{Z}_{\odot}\right)$. The dust budget of a galaxy of this size is also likely dominated by only a few sources, highlighting the need to understand how efficiently they produce dust, as well as the composition of the dust that is produced.

Support for program HST-GO-14845 was provided by NASA through a grant from the Space Telescope Science Institute. STScI is operated by the Association of Universities for Research in Astronomy, Inc. under NASA contract NAS 5-26555. This work is based in part on observations made with the Spitzer Space Telescope, which is operated by the Jet Propulsion Laboratory, California Institute of Technology under a contract with NASA. I.M. and A.A.Z. acknowledge support from the UK Science and Technology Facility Council under grants ST/L000768/1 and ST/P000649/1. This research has also made use of the Vizier catalog access tool, CDS, Strasbourg, France. This research has made use of the Dusty Evolved Star Kit (DESK; https://github.com/s-goldman/ Dusty-Evolved-Star-Kit).

Facilities: Spitzer Space Telescope, Hubble Space Telescope.
Software: the DESK, MATCH (Dolphin 2002), DUSTiNGS pipeline (Boyer et al. 2015a), DOLPHOT (Dolphin 2000).

\section{Appendix Galaxies}

After classifying our AGB candidates using color-color cuts, the HST image revealed that several sources were not clear point sources. These are sources above the TRGB, but that were determined to be either K-type giants or foreground stars $(\mathrm{K} / \mathrm{FG})$, extended sources or background galaxies (Ext), or sources that did not meet our sharpness or crowding cutoffs.

1. Several sources that were initially classified as AGB stars in Lee (2016) have now been reclassified as extended sources as a result of the higher-resolution imaging (Table 2; Figure 9).

2. AGB candidates that met our TRGB criteria and color, sharpness, and crowding cuts, but not our image quality criteria, are shown in Table 3 (extended objects are shown in Figure 8).

3. Table 3 shows the photometry for sources that we have classified or reclassified as foreground stars or extended sources.

There are four sources that fall in the "foreground" region of our color-color diagram in Figure 3 that are not listed as foreground stars in Table 3. Three of these are listed in Table 2, as they were previously categorized as AGB candidates, and one was below the TRGB and has been found to be a galaxy (Source 113).

Table 3

Leo P Additional Photometry

\begin{tabular}{|c|c|c|c|c|c|c|}
\hline ID & $\begin{array}{l}\text { R.A. } \\
\text { (J2000) }\end{array}$ & $\begin{array}{l}\text { Decl. } \\
\text { (J2000) }\end{array}$ & $\begin{array}{c}\text { F127M } \\
(1.27 \mu \mathrm{m})\end{array}$ & $\begin{array}{c}\text { F139M } \\
(1.39)\end{array}$ & $\begin{array}{c}\mathrm{F} 153 \mathrm{M} \\
(1.53)\end{array}$ & Type \\
\hline 23 & 155.4360246 & 18.0828429 & 13.80 & 13.66 & 13.54 & $\mathrm{~K} / \mathrm{FG}$ \\
\hline 24 & 155.4305265 & 18.0842080 & 15.74 & 15.56 & 15.35 & $\mathrm{~K} / \mathrm{FG}$ \\
\hline 25 & 155.4256185 & 18.0944481 & 16.04 & 15.89 & 15.69 & $\mathrm{~K} / \mathrm{FG}$ \\
\hline 26 & 155.4302984 & 18.0870885 & 16.15 & 16.08 & 15.90 & $\mathrm{~K} / \mathrm{FG}$ \\
\hline 27 & 155.4256132 & 18.0943271 & 16.87 & 16.74 & 16.56 & $\mathrm{~K} / \mathrm{FG}$ \\
\hline 28 & 155.4312416 & 18.0860352 & 18.57 & 18.46 & 18.28 & $\mathrm{~K} / \mathrm{FG}$ \\
\hline 29 & 155.4558705 & 18.0853354 & 19.76 & 19.60 & 19.41 & $\mathrm{~K} / \mathrm{FG}$ \\
\hline 31 & 155.4268835 & 18.0764639 & 21.35 & 21.22 & 21.16 & $\mathrm{~K} / \mathrm{FG}$ \\
\hline 45 & 155.4296613 & 18.0882035 & 20.55 & 20.23 & 19.95 & $\ldots$ \\
\hline 52 & 155.4450924 & 18.0902926 & 20.71 & 20.56 & 20.13 & Ext \\
\hline 98 & 155.4289005 & 18.1027023 & 21.40 & 20.92 & 20.77 & Ext \\
\hline 211 & 155.4403415 & 18.1010466 & 21.78 & 21.43 & 21.15 & Ext \\
\hline 494 & 155.4186578 & 18.0772001 & 22.72 & 22.68 & 21.36 & $\ldots$ \\
\hline 780 & 155.4254298 & 18.0992646 & 23.94 & 23.83 & 21.67 & $\cdots$ \\
\hline 2009 & 155.4241478 & 18.1089321 & 23.36 & 23.12 & 22.25 & $\ldots$ \\
\hline 2049 & 155.4252101 & 18.0853781 & 24.34 & 22.48 & 22.99 & Ext \\
\hline
\end{tabular}



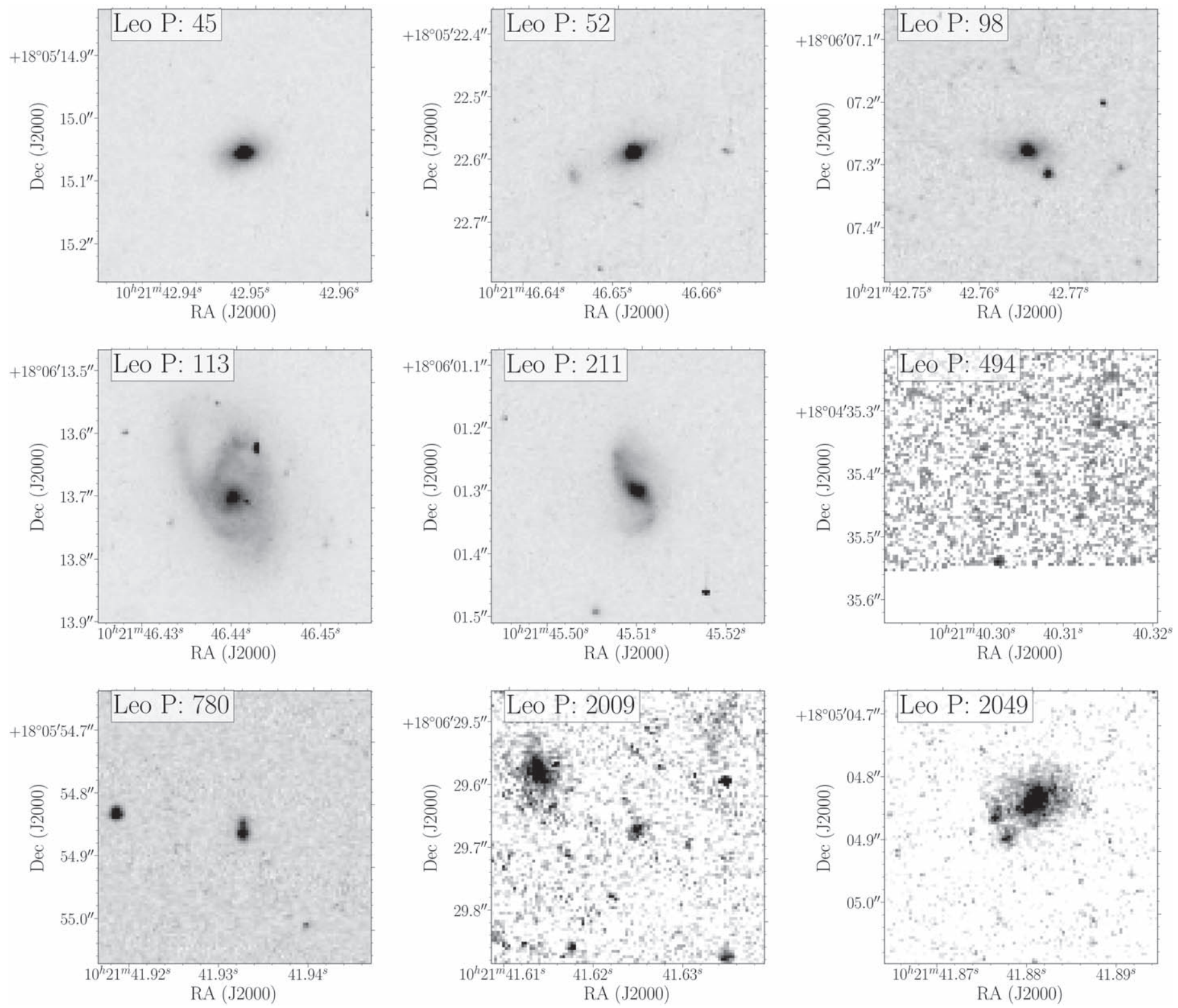

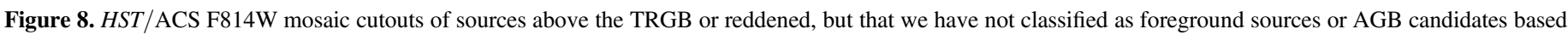

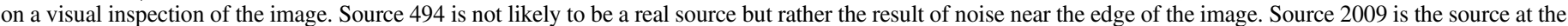
center of the image. 

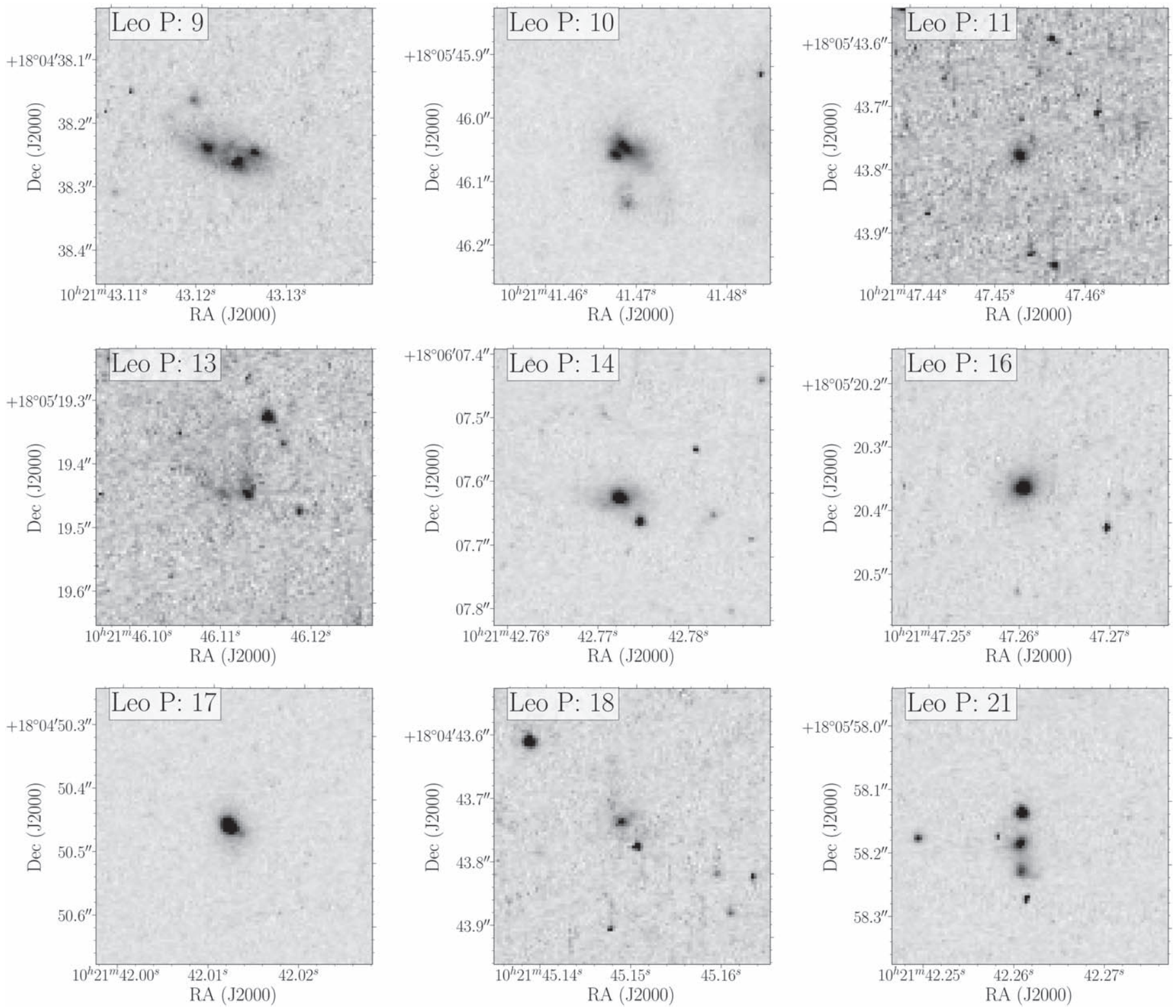


sources.

\section{ORCID iDs}

S. R. Goldman 1 https://orcid.org/0000-0002-8937-3844

M. L. Boyer (1) https://orcid.org/0000-0003-4850-9589

K. B. W. McQuinn (ํ) https://orcid.org/0000-0001-5538-2614

G. C. Sloan (i) https://orcid.org/0000-0003-4520-1044

I. McDonald (1) https://orcid.org/0000-0003-0356-0655

J. Th. van Loon (i) https://orcid.org/0000-0002-1272-3017

A. A. Zijlstra (1) https://orcid.org/0000-0002-3171-5469

A. S. Hirschauer (10 https://orcid.org/0000-0002-2954-8622

E. D. Skillman (1) https://orcid.org/0000-0003-0605-8732

\section{References}

Aoki, W., Tsuji, T., \& Ohnaka, K. 1999, A\&A, 350, 945

Aringer, B., Girardi, L., Nowotny, W., Marigo, P., \& Bressan, A. 2016, MNRAS, 457, 3611

Aringer, B., Girardi, L., Nowotny, W., Marigo, P., \& Lederer, M. T. 2009, A\&A, 503, 913
Bacon, R., Accardo, M., Adjali, L., et al. 2010, Proc. SPIE, 7735, 773508 Battinelli, P., \& Demers, S. 2008, A\&A, 493, 1075

Battinelli, P., Demers, S., \& Mannucci, F. 2007, A\&A, 474, 35

Beelen, A., Cox, P., \& Benford, D. J. 2006, ApJ, 642, 694

Bellazzini, M., Beccari, G., Fraternali, F., et al. 2014, A\&A, 566, A44

Berg, D. A., Skillman, E. D., Marble, A. R., et al. 2012, ApJ, 754, 98

Bernstein-Cooper, E. Z., Cannon, J. M., Elson, E. C., et al. 2014, AJ, 148, 35

Bertoldi, F., Carilli, C. L., Cox, P., et al. 2003, A\&A, 406, L55

Blum, R. D., Srinivasan, S., Kemper, F., Ling, B., \& Volk, K. 2014, AJ, 148,86

Boothroyd, A. I., Sackmann, I. J., \& Ahern, S. C. 1993, ApJ, 416, 762

Boyer, M. L., Girardi, L., Marigo, P., et al. 2013, ApJ, 774, 83

Boyer, M. L., McQuinn, K. B. W., Barmby, P., et al. 2015a, ApJ, 800, 51

Boyer, M. L., McQuinn, K. B. W., Barmby, P., et al. 2015b, ApJS, 216, 10

Boyer, M. L., McQuinn, K. B. W., Groenewegen, M. A. T., et al. 2017, ApJ, 851,152

Boyer, M. L., Srinivasan, S., van Loon, J. T., et al. 2011, AJ, 142, 103

Boyer, M. L., Williams, B. F., Aringer, B., et al. 2019, ApJ, 879, 109

Boyer, M. L., Woodward, C. E., van Loon, J. T., et al. 2006, AJ, 132, 1415

Cardelli, J. A., Clayton, G. C., \& Mathis, J. S. 1989, ApJ, 345, 245

Cernicharo, J., Yamamura, I., Gonz-Ìalez-Alfonso, E., et al. 1999, ApJL, 526, L41 
Clement, C. M., Muzzin, A., Dufton, Q., et al. 2001, AJ, 122, 2587

Dolphin, A. E. 2000, PASP, 112, 1383

Dolphin, A. E. 2002, MNRAS, 332, 91

El-Badry, K., Wetzel, A., Geha, M., et al. 2016, ApJ, 820, 131

Elitzur, M., \& Ivezić, Ž 2001, MNRAS, 327, 403

Evans, C. J., Castro, N., Gonzalez, O. A., et al. 2019, A\&A, 622, A129

Feast, M., Whitelock, P., \& Menzies, J. 2002, MNRAS, 329, L7

Frogel, J. A., \& Hyland, A. R. 1972, Les Spectres des Astres dans l'Infrarouge et les Microondes, 111

Giovanelli, R., Haynes, M. P., Kent, B. R., et al. 2005, AJ, 130, 2598

Goldman, S. R., Boyer, M. L., Mc Quinn, K. B. W., et al. 2019, ApJ, 877, 49

Goldman, S. R., van Loon, J. T., Zijlstra, A. A., et al. 2017, MNRAS, 465, 403

Hanner, M. S. 1988, Infrared observations of comets Halley and Wilson and properties of the grains. Tech. Rep., (Pasadena, CA: Jet Propulsion Lab., California Institute of Technology)

Herwig, F. 2004, ApJS, 155, 651

Hirschauer, A. S., Salzer, J. J., Skillman, E. D., et al. 2016, ApJ, 822, 108

Hodapp, K. W., Jensen, J. B., Irwin, E. M., et al. 2003, PASP, 115, 1388

Höfner, S., \& Olofsson, H. 2018, A\&ARv, 26, 1

Jones, O. C., Maclay, M. T., Boyer, M. L., et al. 2018, ApJ, 854, 117

Jones, O. C., Woods, P. M., Kemper, F., et al. 2017, MNRAS, 470, 3250

Karambelkar, V. R., Adams, S. M., Whitelock, P. A., et al. 2019, ApJ, 877, 110

Kasliwal, M. M., Bally, J., Masci, F., et al. 2017, ApJ, 839, 88

Kraemer, K. E., Sloan, G. C., Price, S. D., \& Walker, H. J. 2002, ApJS, 140,389

Lagadec, E., \& Zijlstra, A. A. 2008, MNRAS, 390, L59

Lakićević, M., van Loon, J. T., Meixner, M., et al. 2015, ApJ, 799, 50

Lebzelter, T., \& Wood, P. R. 2005, A\&A, 441, 1117

Lee, C.-H. 2016, MNRAS, 461, L37

Lee, H., Skillman, E. D., Cannon, J. M., et al. 2006, ApJ, 647, 970

Levesque, E. M., Massey, P., Olsen, K. A. G., et al. 2006, ApJ, 645, 1102

Lorenz, D., Lebzelter, T., Nowotny, W., et al. 2011, A\&A, 532, 78

Marigo, P., Girardi, L., Bressan, A., et al. 2017, ApJ, 835, 77

Marshall, J. R., van Loon, J. T., Matsuura, M., et al. 2004, MNRAS, 355, 1348

Massey, P., Levesque, E. M., Olsen, K. A. G., Plez, B., \& Skiff, B. A. 2007, ApJ, 660, 301

Matsunaga, N. \& IRSF/SIRIUS Team 2007, in ASP Conf. Ser. 378, Why Galaxies Care About AGB Stars: Their Importance as Actors and Probes, ed. F. Kerschbaum, C. Charbonnel, \& R. F. Wing (San Francisco, CA: ASP), 86

McConnachie, A. W. 2012, AJ, 144, 4

McDonald, I., Boyer, M. L., van Loon, J. T., et al. 2011b, ApJS, 193, 23

McDonald, I., Boyer, M. L., van Loon, J. T., \& Zijlstra, A. A. 2011a, ApJ, 730, 71

McDonald, I., van Loon, J. T., Decin, L., et al. 2009, MNRAS, 394, 831

McDonald, I., van Loon, J. T., Sloan, G. C., et al. 2011c, MNRAS, 417, 20

McDonald, I., White, J. R., Zijlstra, A. A., et al. 2012, MNRAS, 427, 2647
McQuinn, K. B. W., Boyer, M. L., Mitchell, M. B., et al. 2017, ApJ, 834, 78 McQuinn, K. B. W., Skillman, E. D., Berg, D., et al. 2013, AJ, 146, 145

McQuinn, K. B. W., Skillman, E. D., Dolphin, A., et al. 2015a, ApJ, 812, 158 McQuinn, K. B. W., Skillman, E. D., Dolphin, A., et al. 2015b, ApJL, 815, L17

Menzies, J. W., Whitelock, P. A., \& Feast, M. W. 2015, MNRAS, 452, 910

Menzies, J. W., Whitelock, P. A., Feast, M. W., \& Matsunaga, N. 2010, MNRAS, 406, 86

Menzies, J. W., Feast, M. W., Whitelock, P. A., \& Matsunaga, N. 2011, MNRAS, 414, 3492

Menzies, J., Feast, M., Whitelock, P., et al. 2008, MNRAS, 385, 1045

Nanni, A., Bressan, A., Marigo, P., Girardi, L., et al. 2013, MNRAS, 434, 2390

Nanni, A., Bressan, A., Marigo, P., \& Girardi, L. 2014, MNRAS, 438, 2328

Nanni, A., Marigo, P., Girardi, L., et al. 2018, MNRAS, 473, 5492

Nanni, A., Marigo, P., Groenewegen, M. A. T., et al. 2016, MNRAS, 462, 1215

Origlia, L., Ferraro, F. R., Fusi Pecci, F., \& Rood, R. T. 2002, ApJ, 571, 458

Ossenkopf, V., Henning, T., \& Mathis, J. S. 1992, A\&A, 261, 567

Rafelski, M., Wolfe, A. M., Prochaska, J. X., Neeleman, M., \& Mendez, A. J. 2012, ApJ, 755, 89

Rhode, K. L., Salzer, J. J., Haurberg, N. C., et al. 2013, AJ, 145, 149

Robson, I., Priddey, R. S., Isaak, K. G., \& McMahon, R. G. 2004, MNRAS, 351, L29

Saviane, I., Rizzi, L., Held, E. V., Bresolin, F., \& Momany, Y. 2002, A\&A, 390, 59

Schlafly, E. F., \& Finkbeiner, D. P. 2011, ApJ, 737, 103

Skillman, E. D., \& Kennicutt, R. C., Jr. 1993, ApJ, 411, 655

Skillman, E. D., Salzer, J. J., Berg, D. A., et al. 2013, AJ, 146, 3

Sloan, G. C., Kraemer, K. E., Wood, P. R., et al. 2008, ApJ, 686, 1056

Sloan, G. C., Kraemer, K. E., McDonald, I., et al. 2016, ApJ, 826, 44

Sloan, G. C., Matsuura, M., Zijlstra, A. A., et al. 2009, Sci, 323, 353

Sloan, G. C., Matsunaga, N., Matsuura, M., et al. 2010, ApJ, 719, 1274

Sloan, G. C., Matsuura, M., Lagadec, E., et al. 2012, ApJ, 752, 140

Smith, V. V., Suntzeff, N. B., Cunha, K., et al. 2000, AJ, 119, 1239

Stetson, P. B. 1987, PASP, 99, 191

Temim, T., Dwek, E., Tchernyshyov, K., et al. 2015, ApJ, 799, 158

van Loon, J. T. 2006, in ASP Conf. Ser. 353, Stellar Evolution at Low Metallicity: Mass Loss, Explosions, Cosmology, ed. H. J. G. L. M. Lamers et al. (San Francisco, CA: ASP), 211

van Loon, J. T., Cohen, M., Oliveira, J. M., et al. 2008, A\&A, 487, 1055

Ventura, P., D’Antona, F., \& Mazzitelli, I. 2002, A\&A, 393, 215

Volk, K., Xiong, G.-Z., \& Kwok, S. 2000, ApJ, 530, 408

Whitelock, P. A. 1986, MNRAS, 219, 525

Whitelock, P. A. 2012, Ap\&SS, 341, 123

Whitelock, P. A., Menzies, J. W., Feast, M. W., et al. 2009, MNRAS, 394, 795

Whitelock, P. A., Menzies, J. W., Feast, M. W., \& Marigo, P. 2018, MNRAS, 473,173 